\title{
Nonresonant tunneling in InGaP/InAIP asymmetric double quantum wells
}

\author{
O. Buccafusca, J. L. A. Chilla, C. S. Menoni, J. J. Rocca, M. J. Hafich, L. M. Woods, \\ and G. Y. Robinson \\ Center of Optoelectronic Computing Systems and Department of Electrical Engineering, Colorado State \\ University, Fort Collins, Colorado 80523
}

(Received 6 August 1992; accepted for publication 9 November 1992)

\begin{abstract}
Nonresonant tunneling rates have been measured in InGaP/InAlP asymmetric double quantum-well structures for which optical phonon assisted tunneling is energetically forbidden. For an initial photoexcited carrier density of $2.4 \times 10^{11} \mathrm{~cm}^{-2}$, tunneling times of 220,60 , and less than 9 ps have been measured in samples with barrier thickness $4.5,3.0$, and $1.5 \mathrm{~nm}$, respectively. The tunneling times were found to be strongly dependent on carrier density. The measured tunneling times and their dependence on carrier density are compatible with impurity scattering being the dominant mechanism assisting the tunneling.
\end{abstract}

Tunneling phenomena in semiconductor structures is being actively investigated for fundamental reasons and possible device applications. ${ }^{1,2}$ Recently, resonant and nonresonant carrier tunneling has been studied in $\mathrm{GaAs}$ based asymmetric double quantum-well (ADQW) structures $^{3-11}$ in part motivated by their potential use in optical switching and computing. ${ }^{9,10}$ Herein, we report the first tunneling measurements in InGaP/InAlP asymmetric double quantum wells which are suitable for the design of modulators that operate in the visible region of the spectrum. In this work, nonresonant carrier tunneling was investigated in ADQW structures where optical phonon assisted tunneling is energetically forbidden. Steady state and time resolved photoluminescence (PL) measurements were conducted on samples with different barrier thicknesses as a function of carrier density and temperature.

The samples studied, grown by gas source MBE, were unintentionally $n$ doped and consisted of 15 periods of ADQW structures separated by an InAlP layer of $15 \mathrm{~nm}$. Each period consists of InGaP wells of 6 and $12 \mathrm{~nm}$ widths, separated by an InAlP barrier having a different width for each sample: $1.5,3.0,4.5$, and $15 \mathrm{~nm}$. The layer thicknesses are nominal values with an error of less than $10 \%$. The structure with a $15 \mathrm{~nm}$ barrier, for which no carrier tunneling is expected to occur, was used as a control sample.

The epitaxial structures were grown on (100) $n+\mathrm{GaAs}$ substrates at approximately $1 \mu \mathrm{m} / \mathrm{h}$ at $500^{\circ} \mathrm{C}$ using thermally cracked $\mathrm{AsH}_{3}$ and $\mathrm{PH}_{3}$, conditions that have previously been shown to produce abrupt interfaces and high quality quantum wells. ${ }^{12,13}$ Each sample consisted of a GaAs layer ( $0.25 \mu \mathrm{m}$ thickness) followed by 15 periods of the ADQW structure and capped by an InAIP layer (0.5 $\mu \mathrm{m}$ thickness). At each heterointerface of the $A D Q W s$, the growth was paused for $18 \mathrm{~s}$ without interruption of the $\mathrm{PH}_{3}$ flow. X-ray diffraction indicated the alloy compositions to be $\operatorname{In}_{0.48} \mathrm{Ga}_{0.52} \mathbf{P}$ and $\operatorname{In}_{0.47} \mathrm{Al}_{0.53} \mathrm{P}$, with lattice match to the GaAs substrate to within 500 ppm. Previous measurements indicate an impurity concentration of $(2-5) \times 10^{16} \mathrm{~cm}^{-3}$ in the InGaP wells. ${ }^{13}$

In the PL measurements, the samples were excited by a train of 2 ps pulses produced by a Rhodamine 560 dye laser synchronously pumped by a frequency doubled mode-locked Nd-YLF laser at a repetition rate of $76 \mathrm{MHz}$. The excitation energy, around $2.14 \mathrm{eV}$, was selected to excite both wells simultaneously and to be sufficiently separated from the photon energies corresponding to the PL of the two quantum wells to facilitate detection. The laser beam was focused on the sample with a spot diameter of approximately $36 \mu \mathrm{m}$. Steady state PL spectra were taken with a $0.75 \mathrm{~m}$ focal length spectrometer and a Hamamatsu R2228 photomultiplier using standard lock-in techniques. The time resolved PL was measured by a synchroscan streak cameras (Hamamatsu M1955) coupled to a thermoelectrically cooled two-dimensional $\mathrm{CCD}$ array detector. The streak camera was attached to the exit of a $0.3 \mathrm{~m}$ focal length spectrometer provided with a $150 \mathrm{~g} / \mathrm{mm}$ grating. The time resolution of the system was determined to be 9 ps. The samples were mounted in a closed cycle refrigerated cryostat for low-temperature measurements.

Figure 1 shows the steady state PL signal corresponding to the four $A D Q W$ structures with different barrier thicknesses obtained at $11 \mathrm{~K}$. All spectra show two peaks: the stronger one, at lower energy, corresponds to radiative recombination in the wide well and the weaker peak, at higher energy, to the same process in the narrow well. The decreasing PL intensity for the narrow well with decreasing barrier thickness suggests that carriers tunnel from the narrow well to the wide well. This is confirmed by the time resolved PL measurements which, as discussed below, show a strong dependence of the time decay of the narrow well PL with barrier thickness.

From the steady state PL data of Fig. 1 and the values for effective masses, the band gap from 'Ref. 13 and the band offset given in Ref. 14, we estimated the energy difference between levels in the wells. For all the samples in which tunneling can occur, these calculations yield a difference between the first energy levels in the conduction band of the two wells of less than $34 \mathrm{meV}$. This energy difference is more than $10 \mathrm{meV}$ below the LO phonon energy for InGaP having the same composition as in our samples. ${ }^{15}$ This implies that, in these samples, LO phonon emission is not energetically available to assist the tunneling process. Next, we discuss the results of time resolved PL measurements.

Time resolved PL signals obtained at $11 \mathrm{~K}$ are shown 


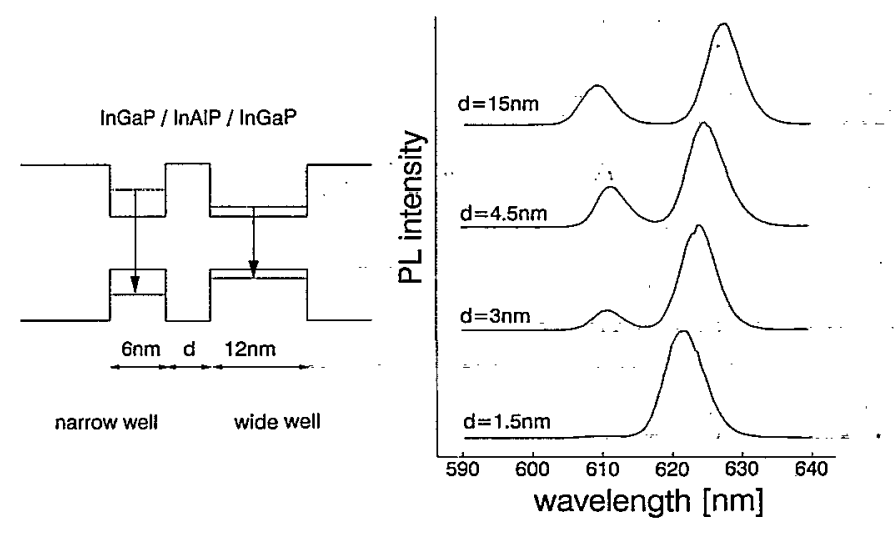

FIG. 1. CW PL at $11 \mathrm{~K}$ of ADQWs with different barrier thickness, d. A significant drop in the intensity of the PL peak associated with the narrow well is observed when the barrier thickness is reduced.

in Fig. 2 as a function of barrier thickness. Figures 2(a) and 2(b) illustrate the temporal evolution of the PL peak corresponding to the narrow and the wide well, respectively. For the sample with the $15 \mathrm{~nm}$ barrier, the time decay of the PL from the narrow well is only slightly shorter than that corresponding to the wide well, which is about 300 ps. As the barrier thickness is decreased, the decay time of the narrow well PL is observed to be consistently shorter. Values of 130,50 , and less than 9 ps (the time resolution of our system) were measured for the ADQWs with barriers of $4.5,3.0$, and $1.5 \mathrm{~nm}$, respectively. In contrast, the decay time of the wide well PL was found to be independent for all the samples, except for the ADQW with a $1.5 \mathrm{~nm}$ barrier that showed a shorter decay, probably due to a variation in sample quality.

The decay time of the wide well PL is due to both radiative and nonradiative recombination. In the narrow well, however, carriers can also relax through tunneling to the wide well. The tunneling time can then be calculated from the measured values of the narrow well PL decay
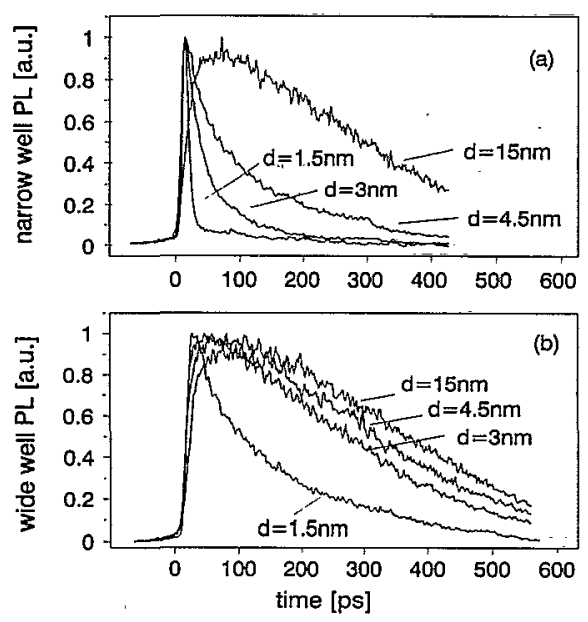

FIG. 2. Time resolved PL signals corresponding to the PL peak wavelength of the narrow well (a) and the wide well (b) for ADQWs with different barrier corresponding to an initial photoexcited sheet carrier density of $2.4 \times 10^{11} \mathrm{~cm}^{-2}$. The measurements were conducted at $11 \mathrm{~K}$.
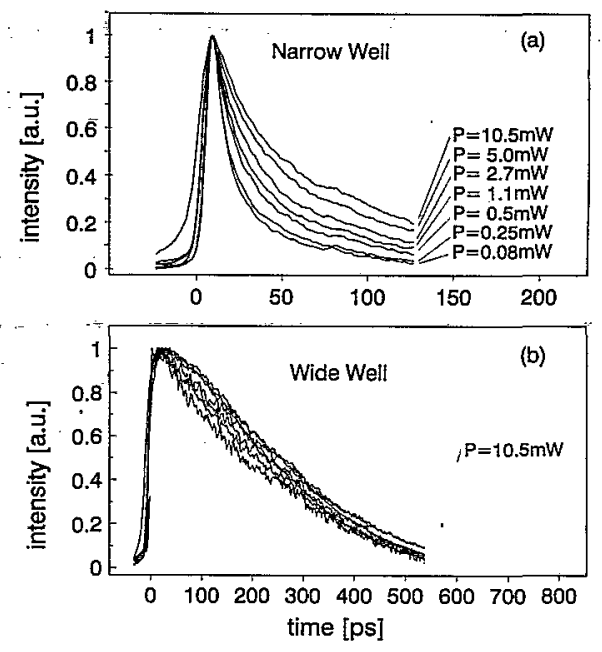

FIG. 3. Low temperature $(11 \mathrm{~K})$ time resolved PL signals for the ADQW with a $3 \mathrm{~nm}$ barrier as a function of excitation intensity for both the narrow well (a) and the wide well (b).

time and the narrow well recombination time. To calculate the narrow well recombination time, we measured the decay rate of the corresponding wide well and corrected this value for the difference in well thickness according to the ratio of the PL decay times from both wells in our control sample. For an initial photoexcited carrier concentration of $2.4 \times 10^{11} \mathrm{~cm}^{-2}$, the tunneling times were calculated to be approximately 220,60 , and less than 9 ps for the samples with $4.5,3.0$ and $1.5 \mathrm{~nm}$ barriers, respectively. These relatively long tunneling times are consistent with the fact that LO phonon assisted tunneling is not available in these samples. Acoustic phonon scattering can also be ruled out as the dominant mechanism assisting the tunneling. Analysis of the time resolved PL spectra shows that the intraband carrier relaxation time due to acoustic phonon scattering is of tens of picoseconds. The associated interwell transfer time is expected to be several orders of magnitude larger due to the reduced overlap of the wave functions.

In the absence of phonon assisted tunneling, the scattering mechanisms that may contribute to tunneling are electron-electron, acoustic phonon, impurity, interfaceroughness, and alloy disorder. To gain additional information on the role of different scattering mechanisms, we measured the tunneling rate dependence on carrier density and temperature. Time dependent PL measurements were performed for laser intensities ranging from 0.08 to 10.5 $\mathrm{mW}$ corresponding to sheet carrier densities between $8 \times 10^{9}$ and $1 \times 10^{12} \mathrm{~cm}^{-2}$. Figure 3 shows the measured dependence of the PL decays for the ADQW with a $3 \mathrm{~nm}$ barrier as a function of excitation intensity. For the wide well, the PL decay was relatively independent of carrier density. In contrast, the narrow well PL decay time increased approximately four times as the intensity was increased in the range mentioned above. A similar, but weaker, dependence on excitation intensity was measured in the narrow well PL decay of the sample with the $4.5 \mathrm{~nm}$ barrier. For the sample with the $1.5 \mathrm{~nm}$ barrier the time decay of the narrow well PL remained below the temporal 
resolution of our detection system for intensities below 6.5 $\mathrm{mW}$ and for intensities above this value the PL decay was also observed to increase. Local heating of the samples caused by the increased intensity may affect PL decay measurements. However, this effect can be ruled out as a possible cause of an increase in carrier lifetime because measurements done as a function of temperature showed the opposite behavior: the PL decay of the wide well was observed to decrease slightly as a function of temperature. Hence, we conclude that the scattering processes that assist tunneling are carrier density dependent.

A possible cause of the observed decrease of the tunneling rate with the increased photoexcited density is space-charge buildup caused by the significantly larger tunneling rates for electrons compared to that of holes, due to their large difference in effective mass. This difference in tunneling rates can cause a charge buildup between the two wells, producing an electric field which increases the potential barrier between the two wells, opposing tunneling. We have calculated that the maximum potential change for our largest carrier density power to be $5 \mathrm{meV}$. In this calculation we assumed the extreme case in which all of the electrons and none of the holes have tunneled. Since, in practice, the change in the potential barrier is much smaller than this limit value, which is small compared with the energy separation between the initial and final states, we conclude that space-charge effects are not responsible for the observed density dependence. An analysis of the shape of the measured PL decay as a function of time for difference excitation intensities also shows no evidence of space-charge effects.

The increase of the tunneling time with carrier density rules out electron-electron scattering a the dominant tunneling mechanism. The remaining three processes have a dependence on carrier density which is compatible with our observations. However, calculated tunneling times due to alloy scattering, using the potential given by Mukhopadhyay and Nag, ${ }^{16}$ resulted in times larger than 1 ns. Tunneling times due to interface roughness, calculated by Ferreira and Bastard, ${ }^{17}$ were found to be between one and two orders of magnitude larger than for impurity scattering when the concentration of scatterers is the same as that of impurities. We have calculated the nonresonant tunneling rate for impurity scattering following the procedure outlined by Ferreira and Bastard. ${ }^{17}$ Assuming a density of impurities of $5 \times 10^{16} \mathrm{~cm}^{-3}$, and that these impurities are localized mostly at the interfaces, we calculated nonresonant electron tunneling times of 450,70 , and 12 ps for barrier widths $4.5,3.0$, and $1.5 \mathrm{~nm}$, respectively. These calculated values are very similar to the measured tunneling times. This suggests that the mechanism assisting the tunneling is likely to be impurity scattering. A similar decrease of the nonresonant tunneling rate as a function of the density of photoexcited carriers was recently observed in GaAs/AlGaAs ADQW's. " The measured tunneling dependence in that experiment was found to be compatible with impurity assisted interwell scattering, and it was also suggested that excitons may tunnel as a single entity.

In summary, we have measured for the first time nonresonant tunneling times in InGaP/InAlP ADQW in which LO phonon assisted tunneling is not allowed. The tunneling times were found to increase as a function of the photoexcited carrier density. The magnitude of the tunneling times and their dependence on carrier density are compatible with impurity scattering being the dominant tunneling mechanism.

The authors wish to acknowledge Professor J. Mahan for lending the closed cycle refrigeration system, Dr. D. Patel and M. Prasad for their assistance in the experiment, and Dr. K. Bhattacharyya for helpful discussions. This work was supported by the Center for Optoelectronic Computer Systems through National Science Foundation Grant ECD 9015128 and by the Colorado Advanced Technology Institute. C. S. Menoni acknowledges here support by the Engineering Foundation AFOSR Research Initiation Grant RI-B-91-16.

${ }^{1}$ F. Capasso, K. Mohammed, and A. Y. Cho, IEEE J. Quantum Electron. QE-22, 1853 (1986).

${ }^{2}$ S. Luryi, Superlattices Microstructures 5, 475 (1989).

${ }^{3}$ T. Tada, A. Yamagushi, T. Ninomiya, H. Uchiki, T. Kobayashi, and T. Yao, J. Appl. Phys. 63, 5491 (1988).

${ }^{4}$ S. Muto, T. Inata, A. Tacheuchi, Y. Sugiyama, and T. Fujii, Appl. Phys. Lett. 58, 2393 (1991).

${ }^{5}$ M. Nido, M. G. W. Alexander, W. W. Ruhle, T. Schweizer, and K. Kohler, Appl. Phys. Lett. 56, 355 (1990).

${ }^{6}$ D. Y. Oberli, J. Shah, T. C. Damen, C. W. Tu, T. Y. Chang, D. A. B. Miller, J. E. Henry, R. F. Kopf, N. Sauer, and A. E. DiGiovanni, Phys. Rev. B 40, 3028 (1989).

${ }^{7}$ K. Leo, J. Shah, E. O. Gobel, T. C.Damen, S. Schmitt-Rink, W. Schafer, and K. Kohler, Phys. Rev. Lett. 66, 201 (1991).

${ }^{8}$ N. Sawaki, R. A. Hopfel, E. Gornik, and H. Kano, Appl. Phys. Lett. 55, 1996 (1989).

${ }^{9}$ C. Tanguy, B. Deveaud, A. Regreny, D. Hulin, and A. Antonetti, Appl. Phys. Lett. 58, 1283 (1991).

${ }^{10}$ A. Tackeuchi, S. Muto, T. Inata, and T. Fujii, Appl. Phys. Lett. 58, 1670 (1991).

"D. H. Levi, D. R. Wake, M. V. Klein, S. Kumar, and H. Morkoç, Phys. Rev. B 45, 4274 (1992).

${ }^{12}$ M. J. Hafich, J. H. Quigley, R. E. Owens, G. Y. Robinson, D. Li, and N. Otsuka, Appl. Phys. Lett. 54, 2686 (1989).

${ }^{13}$ M. J. Hafich, H. Y. Lee, G. Y. Robinson, D. Li, and N. Otsuka, J. Appl. Phys. 69, 752 (1991).

${ }^{14}$ M. O. Watanabe and Y. Ohba, Appl. Phys. Lett. 50, 906 (1987).

${ }^{15}$ M. Zachau and W. T. Masselink, Appl. Phys. Lett. 60, 2098 (1992).

${ }^{16} \mathrm{~S}$. Mukhopadhyay and B. R. Nag, Appl. Phys. Lett. 60, 2897 (1992)

${ }^{17}$ R. Ferreira and G. Bastard, Phys. Rev. B 40, 1074 (1989). 